\title{
Neurotecoma na axila causando dor persistente no ombro: Relato de caso*
}

\section{Neurothekeoma in the Axilla Causing Persistent Shoulder Pain: Case Report}

\author{
Christine Maria Muniz Silva1(1) João Paulo Uchoa Fontenele ${ }^{2(0)}$ Jailson Rodrigues Lopes ${ }^{3}$ (1) \\ Gabriella Cristina Coelho de Brito ${ }^{10}$ Manuel Joaquim Diógenes Teixeira ${ }^{10}$ \\ Francisco Airton Castro Rocha ${ }^{4}{ }^{\circ}$
}

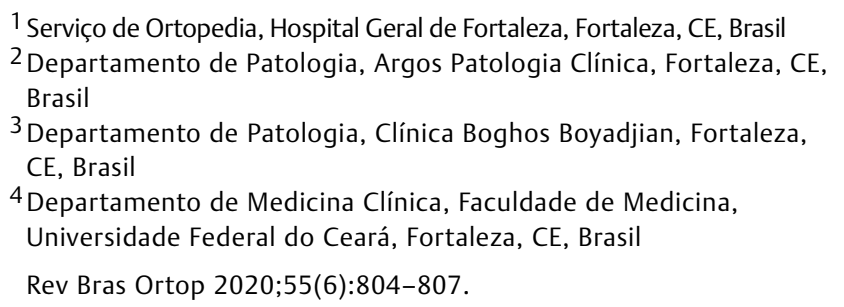

Endereço para correspondência Christine Maria Muniz Silva, MD, MSc, Instituto de Biomedicina, Laboratório de Investigação em Osteoartropatias, Rua Cel. Nunes de Melo, 1315, $1^{\circ}$ Andar, Rodolfo Teófilo, Fortaleza, CE, Brasil (e-mail: arocha@ufc.br).

\section{Resumo \\ Palavras-chave \\ - neurotecoma \\ - ombro \\ - tumor}

\section{Abstract}

Estudo desenvolvido no Serviço de Ortopedia do Hospital Geral de Fortaleza, Fortaleza, CE, Brasil.

recebido

23 de Outubro de 2019

aceito

02 de Março de 2020
Neurotecomas, também conhecidos como mixomas da bainha neural, são tumores benignos raros da bainha neural afetando mais comumente a cabeça, braços e ombros de mulheres entre 20 e 40 anos de idade. Devido à baixa prevalência e quadro clínico mal definido, essas lesões são raramente consideradas no diagnóstico diferencial de tumores cutâneos. Relatamos o caso de uma mulher de 24 anos de idade que procurou atendimento em 2016 relatando dor moderada por mais de um ano e limitação dos movimentos do ombro esquerdo. Ao exame, foi constatada restrição da mobilidade dessa articulação e uma ressonância magnética revelou imagem multilobular com aumento de sinal em T2 na região quadrilateral, aparentando invasão da região cortical do úmero subjacente. A histopatologia de uma biópsia incisional mostrou lesão composta por tecido conjuntivo frouxo, sem sinais de invasão, figuras de mitose ou atipias. Foi realizada excisão completa da lesão e o diagnóstico de neurotecoma foi confirmado após análise histopatológica que incluiu painel imunohistoquímico. À revisão de 18 meses, a paciente estava assintomática com recuperação completa do movimento e sem evidência de recidiva da lesão.

Neurothekeomas, also known as neural sheath myxomas, are rare benign tumors of the neural sheath affecting most commonly the head, arms and shoulder of women in their $2^{\text {nd }}$ and $3^{\text {rd }}$ decades of life. Due to the low prevalence and undefined clinical picture, they are hardly considered in the initial differential diagnosis of skin tumors. We report the case of a 24 year-old woman who was seen in 2016 reporting $>1$ year of moderate pain and limited mobility of her left shoulder. Clinical evaluation revealed restricted mobility of the affected shoulder and nuclear magnetic resonance imaging showed a 


\section{Keywords}

- neurothekeoma

- shoulder

- tumors

T2-weighted contrast-enhanced multilobular mass in the quadrilateral area apparently invading the adjacent humeral cortical region. Histopathology of a needle sample material revealed loose fibroconnective tissue with no signs of invasion, mitosis or atypical figures. Successful surgical excision was performed and the diagnosis of neurothekeoma was confirmed after detailed histopathology, including immunohistochemistry. The patient was asymptomatic at 18 months of follow-up, with full recovery of shoulder movement and no signs of relapse.

\section{Introdução}

O neurotecoma é um raro tumor da bainha neural que acomete a pele, também conhecido como mixoma da bainha neural. Origina-se do endoneuro de nervos periféricos, sendo caracterizado por abundante matriz mucoide. Devido à sua raridade e caráter frequentemente não invasivo, os neurotecomas dificilmente são lembrados como possibilidade diagnóstica, o que nos motivou a preparar o presente relato de caso. ${ }^{1}$ Habitualmente, apresentamse como nódulos fibroelásticos localizados na pele, em regiões da cabeça, pescoço e membros superiores. ${ }^{1,2}$

\section{Relato do Caso}

A paciente tinha 24 anos quando procurou atendimento em agosto de 2016, com queixa de dor moderada, que havia se agravado nos 2 últimos meses, e restrição progressiva da amplitude de movimento em seu ombro esquerdo, ao longo de 1 ano. Não havia história de movimentos repetitivos ou trauma local, bem como queixa de manifestações sistêmicas. A história familiar era negativa para acometimentos musculoesqueléticos relevantes e não havia comorbidades. Fazia uso de analgésicos diariamente, por conta própria. Ao exame físico do ombro esquerdo, encontrava-se leve limitação da rotação externa, ativamente atingindo $60^{\circ}$, mantendo eleva- ção no plano da escápula e rotação interna normais. Os testes para avaliação do manguito rotador foram normais e o exame neurovascular era normal. 0 estudo radiográfico do ombro acometido foi normal ( - Figura 1 ) e a ressonância nuclear magnética (RNM) revelou massa lobular sólida, inespecífica $(3,3 \times 2,6 \times 1,7 \mathrm{~cm})$, ocupando o espaço quadrilateral, adjacente à cortical umeral, com aparente impressão de invasividade ( - Figura 2). Uma cintilografia óssea não mostrou captação na área da lesão.

Foi realizada biópsia incisional, cuja análise se mostrou inconclusiva, sendo indicada ressecção completa da lesão, que foi realizada em abril de 2017. A análise histopatológica do material ressecado, após coloração por HematoxilinaEosina (H\&E), revelou células em formato fusiforme com arranjo estoriforme, sem figuras de atipia, formando nódulos com estroma de colágeno, de aspecto plexiforme, e alguns focos epitelióides, sendo impossível definir a histogênese (-Figura 3).

A imunohistoquímica para proteína S100 e para o antígeno epitelial de membrana (EMA) foi positiva em áreas de proliferação de células fusiformes, associada à positividade para marcação do antígeno CD68 e para expressão de actina de músculo liso (SMA). O índice de proliferação celular, avaliado pela marcação com o antígeno $\mathrm{K}_{\mathrm{i}}-67$, foi $<1 \%$. A análise dos dados clínicos e de imagem, associados à
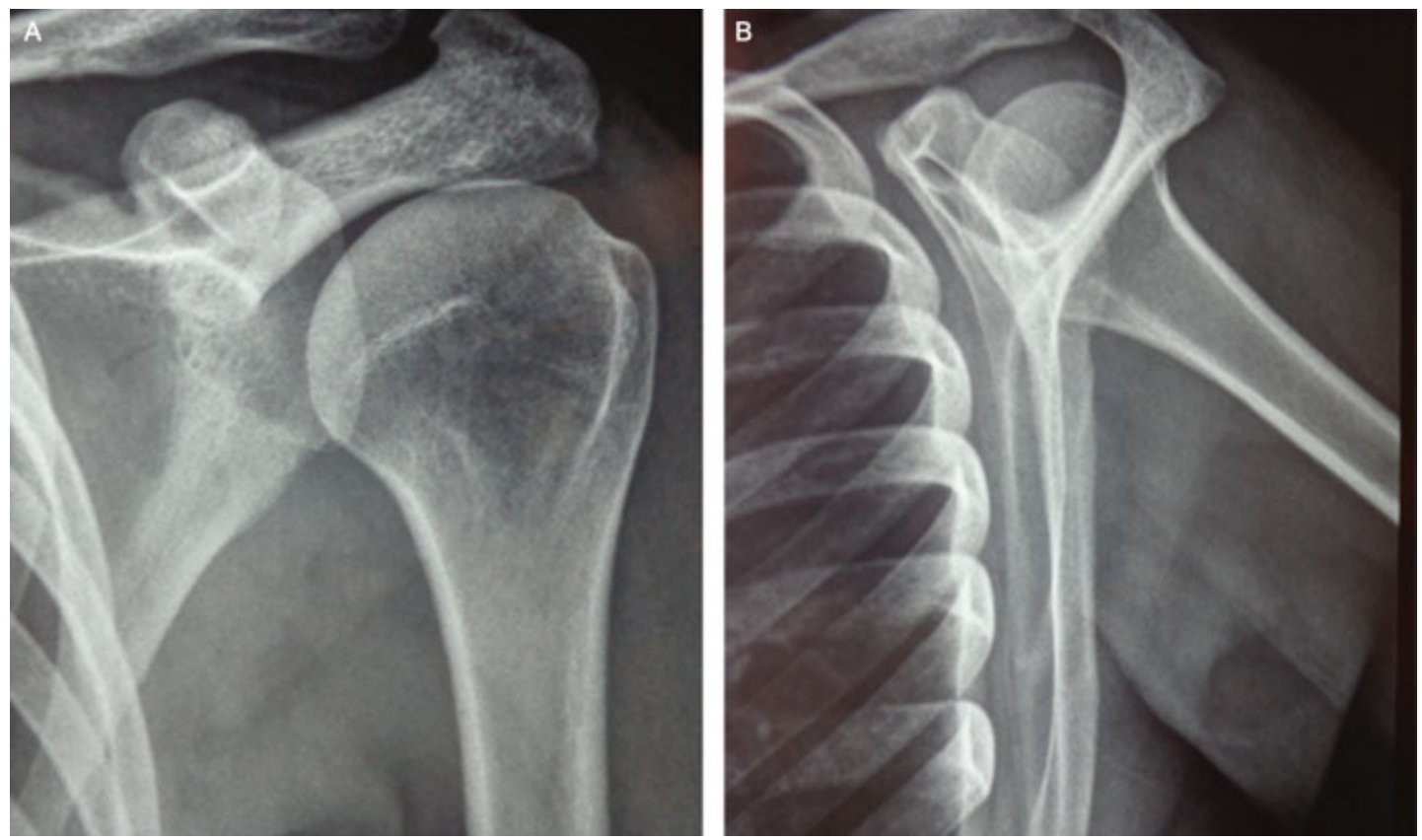

Fig. 1 Radiografias pré-cirúrgicas em posição anteroposterior (A) do ombro e perfil (B) da escápula esquerda. 

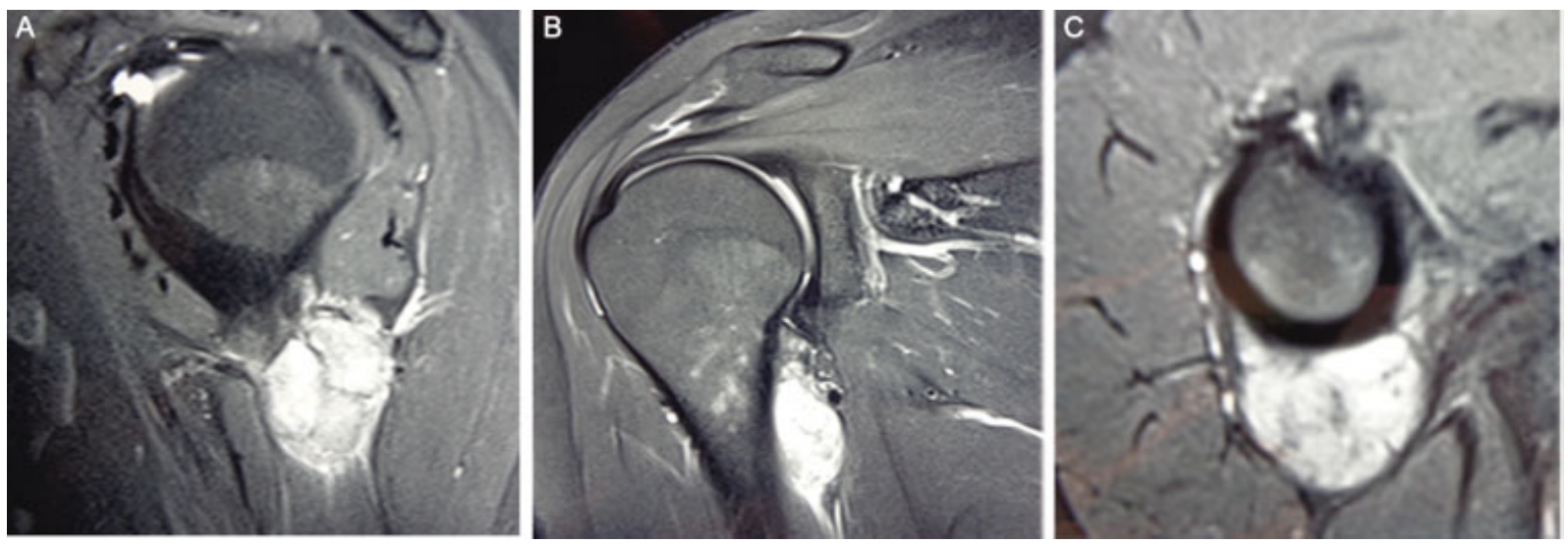

Fig. 2 Imagem de ressonância nuclear magnética do ombro esquerdo em T2 em posição sagital (A), coronal oblíqua (B) e axial com supressão de gordura (C) mostrando massa lobular com septações internas, de contornos bem definidos, sem sinais de invasão, na região proximal da diáfise humeral.

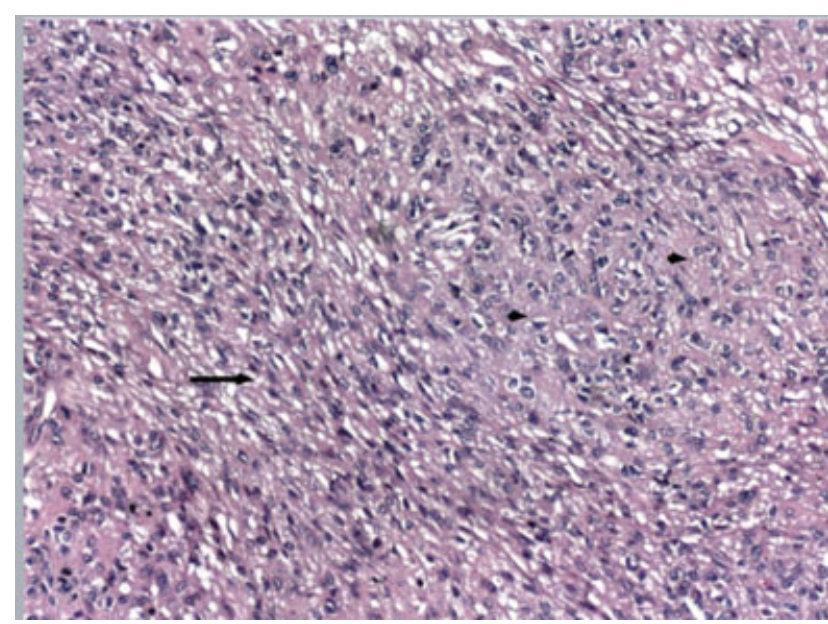

Fig. 3 Aspecto histológico da massa excisada, mostrando predominância de células fusiformes (seta) e outras com aspecto epitelióide (cabeça de seta); não há sinais de atipia ou de figuras mitóticas (coloração por H\&E; Original x100).

avaliação histopatológica, levaram à conclusão diagnóstica de se tratar de neurotecoma.

Após 18 meses do procedimento cirúrgico realizado, a paciente estava assintomática, sem limitação dos movimentos do ombro esquerdo, e uma RNM da região acometida não mostrou recidiva da lesão (- Figura 4).

\section{Histopatologia}

O exame histopatológico foi realizado na rotina, com o material de ressecção sendo incluído em parafina, seguindo-se coloração de cortes de 4- $\mu$ m por H\&E. A análise da imunohistoquímica foi realizada usando aparelho Ventana Benchmark (Ventana Medical Systems, Tucson, AZ, EUA) após desparafinização com tampão EZprep (Ventana Medical Systems, Tucson, AZ, EUA) (04 minutos). A recuperação antigênica foi feita com tampão Cell Conditioning (Ventana Medical Systems, Tucson, AZ, EUA) (30 minutos; pH 8.4) seguido por banho com tampão de reação e amplificação com reagentes UltraView, Hematoxilina e Bluing (Ventana Medical Systems, Tucson, AZ, EUA). Foi utilizado o seguinte painel de anticorpos primários na análise imunohistoquímica: S100 (Clone S1/61/ 69 1:1000; Leica Biosystems, Buffalo Grove, IL, EUA), EMA(E29
1:5000; CellMarque, Darmstadt, Alemanha), CD34 (QBEnd/10 Ready-to-use; Roche, São Paulo, SP, Brasil), CD68 (514h12 Ready-to-use; Roche, São Paulo, SP Brasil), SMA (1A4 1:5000; CellMarque,; Darmstadt, Alemanha) e Ki67 (SP6 1:300; CellMarque, Darmstadt, Alemanha).

\section{Discussão}

Neurotecomas estão tipicamente restritos à pele e tecido subcutâneo, sendo o acometimento do recesso axilar incomum. Mais de 35\% dos neurotecomas estão localizados nos membros superiores $^{2}$ e a idade de acometimento envolve desde crianças de 15 meses de vida até idosos com 84 anos, com média etária de 18 anos de idade. Usualmente, as lesões medem $\sim 1,2 \mathrm{~cm}$ de diâmetro. Trauma local e uso de estrogênio foram propostos como fatores desencadeantes, possivelmente devido à predominância em pacientes do sexo feminino., ${ }^{1,2}$

O aspecto histológico pode ser classificado como mixóide, celular ou misto, baseado, respectivamente, na predominância celular, presença de mucina ou matriz mixóide. A distinção entre neurotecoma e mixomas de bainha neural ainda não está bem estabelecida., ${ }^{3,4}$ Marcadores imunohistoquímicos podem ajudar a discriminar os subtipos dos neurotecomas, tais como: proteína S100, proteína acídica glial(GFAP), receptor do fator de crescimento ceural e antígeno específico de Melanoma ($\left.\mathrm{NKI} / \mathrm{C} 3, \mathrm{~K}_{\mathrm{i}}-\mathrm{M} 1 \mathrm{p}\right)$. A coloração para proteína A6 S100 tem forte marcação no subtipo celular. Histologicamente, os neurotecomas devem ser discriminados de outros tumores fibrohistiocíticos, como os fibromixomas. ${ }^{4-6} \mathrm{~A}$ indicação de ressecção cirúrgica em tumores benignos inclui a localização em áreas que podem restringir a mobilidade e/ou limitar a função. A dor e a limitação progressiva da amplitude de movimento do ombro, bem como a imprecisão do diagnóstico após a análise da biópsia incisional nesse caso justificaram a indicação de excisão cirúrgica. Lesões $>6 \mathrm{~cm}$, envolvendo tecido celular subcutâneo, músculos e vasos sanguíneos, com aspecto celular pleomórfico, são considerados neurotecomas atípicos. Infiltração marginal e alto índice mitótico ( $>3$ por campo de grande aumento) foram associados com o subtipo atípico. Em uma série de 10 pacientes classificados como se apresentando com padrão atípico, seguidos por 5 anos, não houve recidiva local ou 


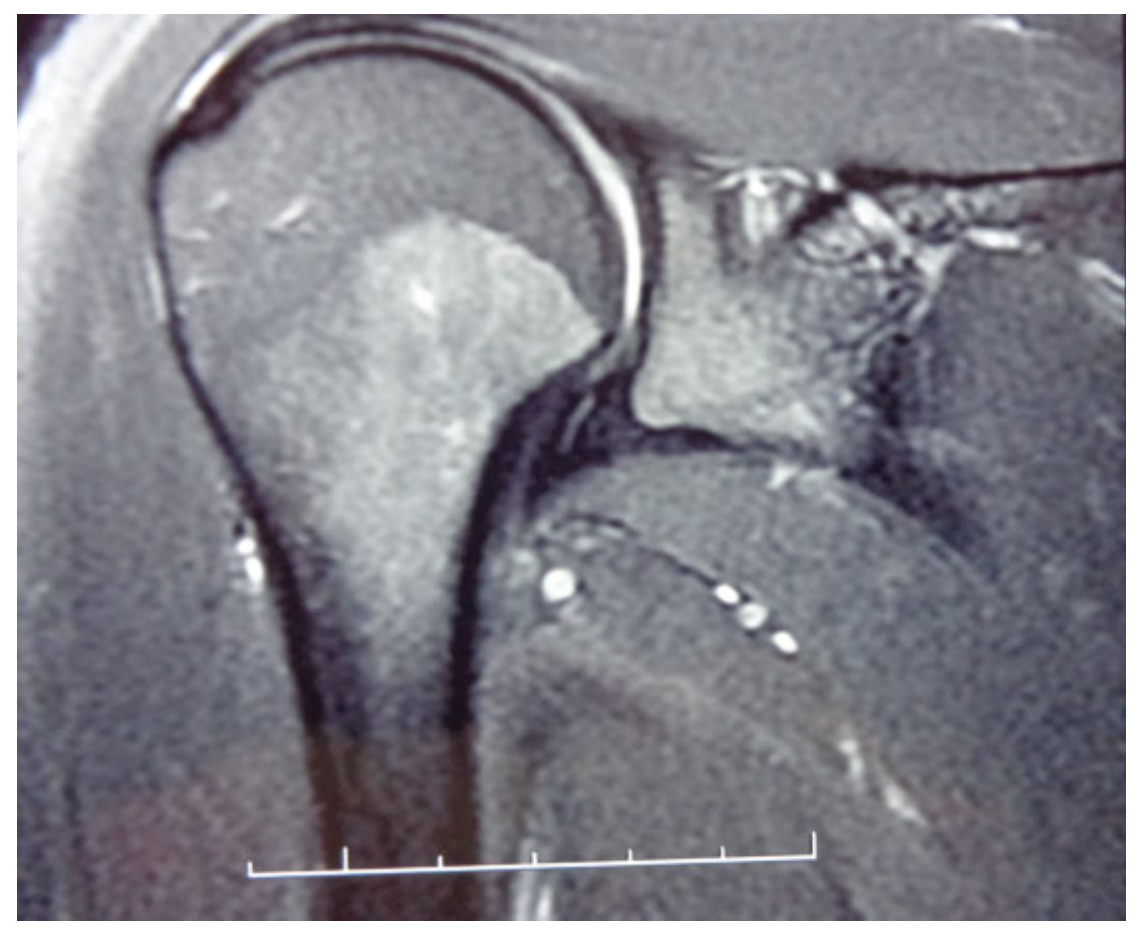

Fig. 4 Aspecto pós-cirúrgico do ombro esquerdo à ressonância nuclear magnética (18 meses), com imagem em T2, em visão coronal.

metástase levando o autor a sugerir que o aparente comportamento histológico agressivo não esteja associado com desfecho clínico. ${ }^{7,8}$

Neurotecomas são difíceis de suspeição antes da análise histopatológica. Não há relato de transformação maligna ou metástase. Pode haver recorrência local, apesar de incomum, particularmente nos tumores classificados como de padrão celular. A excisão cirúrgica completa, com margem de segurança de alguns milímetros, é a abordagem apropriada. Aparentemente, lesões agressivas têm motivado ressecção com margens maiores, mas não há dados a suportar essa abordagem. Os fatores de risco propostos para recidiva do neurotecoma incluem: subtipo mixóide, gênero feminino, localização na cabeça, paciente mais jovem, comprometimento marginal e ausência de tecido adiposo no material ressecado. Presença de células atípicas e número de figuras mitóticas não estão associados com recidiva. ${ }^{1}$

A despeito de serem raros, os neurotecomas precisam ser lembrados como possibilidade diagnóstica em lesões que acometam cabeça e pescoço, posto que a remoção cirúrgica precoce é o tratamento usualmente curativo.

O fato de ter ocorrido no recesso axilar, com queixas relacionadas a dor no ombro, justifica o presente relato de caso, alertando para a necessidade de avançar na investigação por imagem, como realizado. Em casos semelhantes, pode ocorrer atraso no diagnóstico, por confusão com causas mais frequentes de dor no ombro tais como capsulite adesiva ou até doença do manguito rotador, particularmente se considerarmos a dificuldade de acesso à RNM no serviço público. $\mathrm{O}$ planejamento operatório adequado contribuiu para uma intervenção cirúrgica bem-sucedida, de sorte que a paciente se recuperou sem apresentar qualquer sequela funcional.
Conflito de Interesses

Os autores não declaram não haver conflito de interesses.

Agradecimento

Agradecemos ao laboratório Argos Patologia Clínica, Fortaleza, CE, Brasil, 60175-047, pelo processamento histológico.

\section{Referências}

1 Fetsch JF, Laskin WB, Hallman JR, Lupton GP, Miettinen M. Neurothekeoma: an analysis of 178 tumors with detailed immunohistochemical data and long-term patient follow-up information. Am J Surg Pathol 2007;31(07):1103-1114

2 Hornick JL, Fletcher CD. Cellular neurothekeoma: detailed characterization in a series of 133 cases. Am J Surg Pathol 2007;31 (03):329-340

3 Stratton J, Billings SD. Cellular neurothekeoma: analysis of 37 cases emphasizing atypical histologic features. Mod Pathol 2014; 27(05):701-710

4 Fetsch JF, Laskin WB, Miettinen M. Nerve sheath myxoma: a clinicopathologic and immunohistochemical analysis of 57 morphologically distinctive, S-100 protein- and GFAP-positive, myxoid peripheral nerve sheath tumors with a predilection for the extremities and a high local recurrence rate. Am J Surg Pathol 2005;29(12):1615-1624

5 Plaza JA, Torres-Cabala C, Evans H, Diwan AH, Prieto VG. Immunohistochemical expression of S100A6 in cellular neurothekeoma: clinicopathologic and immunohistochemical analysis of 31 cases. Am J Dermatopathol 2009;31(05):419-422

6 Sheth S, Li X, Binder S, Dry SM. Differential gene expression profiles of neurothekeomas and nerve sheath myxomas by microarray analysis. Mod Pathol 2011;24(03):343-354

7 Campanati A, Brandozzi G, Sisti S, Bernardini ML, Offidani AM. Atypical neurothekeoma: a new case and review of the literature. J Cutan Pathol 2007;34(05):435-437

8 Busam KJ, Mentzel T, Colpaert C, Barnhill RL, Fletcher CD. Atypical or worrisome features in cellular neurothekeoma: a study of 10 cases. Am J Surg Pathol 1998;22(09):1067-1072 